\title{
Ambient temperature drying of therapeutic protein solution with use of microwave
}

Tsuruta, T. ${ }^{a^{*}}$; Ogawa, T. ${ }^{\mathbf{b}}$; Abe, R. ${ }^{\text {b; Tanigawa, H. }}{ }^{\mathbf{a}}$

a Faculty. Department of Mechanical Engineering. Kyushu Institute of Technology, Fukuoka, Japan.

${ }^{\mathrm{b}}$ Graduate School of Mechanical Engineering. Kyushu Institute of Technology, Fukuoka, Japan.

*E-mail of the corresponding author: tsuruta.takaharu393@mail.kyutech.jp

\begin{abstract}
High quality drying of therapeutic protein-solution is important in medical and pharmaceutical processing. Freeze-drying is mostly used, but it takes a long drying-time and causes damages of protein structures. In order to improve the drying quality, we propose a microwave vacuum drying performed at ambient temperatures under low-pressure conditions. We are focusing on the Parma-Zyme method for the evaporative drying of protein solutions such as egg white or lysozyme with vitrification. Circular dichroism (CD) spectroscopy is used to detect protein conformation changes due to the drying, and it is found that the ambient temperature drying can preserve the protein conformation.
\end{abstract}

Keywords: Microwave vacuum drying; Freeze-drying; Therapeutic protein; Egg white; Lysozyme 


\section{Introduction}

In recent years, with the progress of biotechnology, the development of pharmaceutical products using biopolymers such as proteins has been rapidly developed. However, it is difficult to stably stored in solution state, many proteins preparations are preserved by freeze-drying (FD). Generally, FD takes a long time (about 1 to 3 days) due to drying accompanied by sublimation of ice, and requires large energy. In addition, changes in the hydration state due to freezing and concentration may result in deactivation and aggregation of proteins. Therefore, studies are under way to prevent denaturation by adding compounds and additives to protein formulations [1]. Considering the situation, we propose a method using a microwave vacuum drying (MVD) as a new method instead of FD. MVD promotes evaporation of water under reduced pressure condition and supplies only latent heat of vaporization by microwave, resulting in the drying at room temperature. It is possible not only to prevent protein damage but also to drastically shorten the dering time. We are focusing on the Perma-Zyme method for the evaporative drying with vitrification, because it has a possibility to increase the vitrification temperature up to the room temperature [2]. Figure 1 shows a solid/liquid state diagram of sucrose water system [3]. In the method of FD, the long process (A-B-C-D) must be followed to get the glassy state for the vital preservation. On the other hand, if the direct phase transition from liquid to solid (glass) at room temperature (A-D), it would have great advantages in the bioindustries. In this study, drying experiments of egg whites and lysozyme protein solutions were carried out using three drying methods of FD, MVD, and a combination of FD and MVD. After the drying, preservation of protein structure/activity, drying time, and solubility were evaluated.

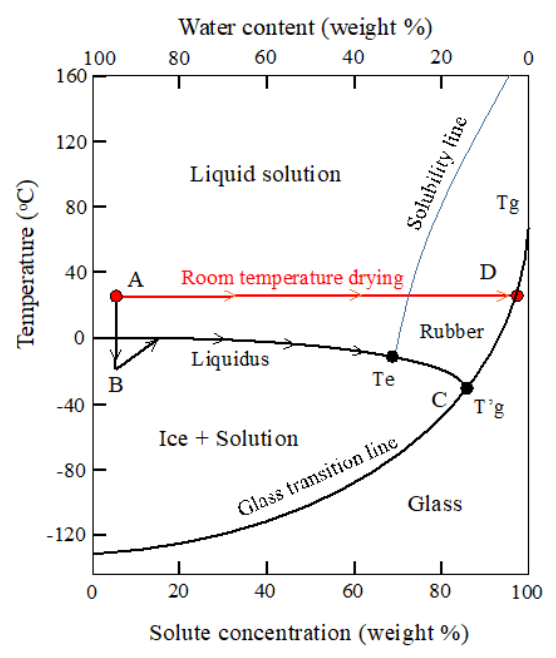

Fig.1 Solid/liquid state diagram of the sucrose water system. 


\section{Experimental Apparatus and Methods}

\subsection{Drying methods}

The experimental apparatus for the microwave vacuum drying is shown in Fig. 2. The drying system consists of the vacuum drying container, vacuum pump, and microwave irradiation equipment $[4,5]$. The drying chamber is a cylinder made from stainless steel, which has an inner diameter of $590 \mathrm{~mm}$ and a height of $345 \mathrm{~mm}$. The microwave is introduced into the chamber from the side. The microwave generator has a magnetron with a $3 \mathrm{~kW}$ irradiation power at $2.45 \mathrm{GHz}$. We can select some intermittent irradiation patterns with a different power as well as a continuous irradiation. In order to irradiate the sample uniformly, a turntable is set at the bottom of the chamber. During the experiment, the samples on the table are rotated at a rate of 3rpm.

As experimental samples, $5 \mathrm{~g}$ of egg whites (albumin) and $5 \mathrm{~g}$ of lysozyme aqueous solution ( $0.5 \mathrm{~g}$ of lysozyme $+4.5 \mathrm{~g}$ of purified water) were used as a model of protein solution. The sample is placed in a PFA Petri dish of $50 \mathrm{~mm}$ in inner diameter. In MVD, four set of samples were placed in a vacuum container, and drying was performed with a microwave output of 50 to $100 \mathrm{~W}$ at a pressure of $20 \mathrm{kPa}$. In FD, the sample was frozen in a freezer at $-25^{\circ} \mathrm{C}$ for more than 12 hours, then the frozen sample was set in a vacuum container and dried at a temperature of $5^{\circ} \mathrm{C}$ under a pressure of $200 \mathrm{~Pa}$.

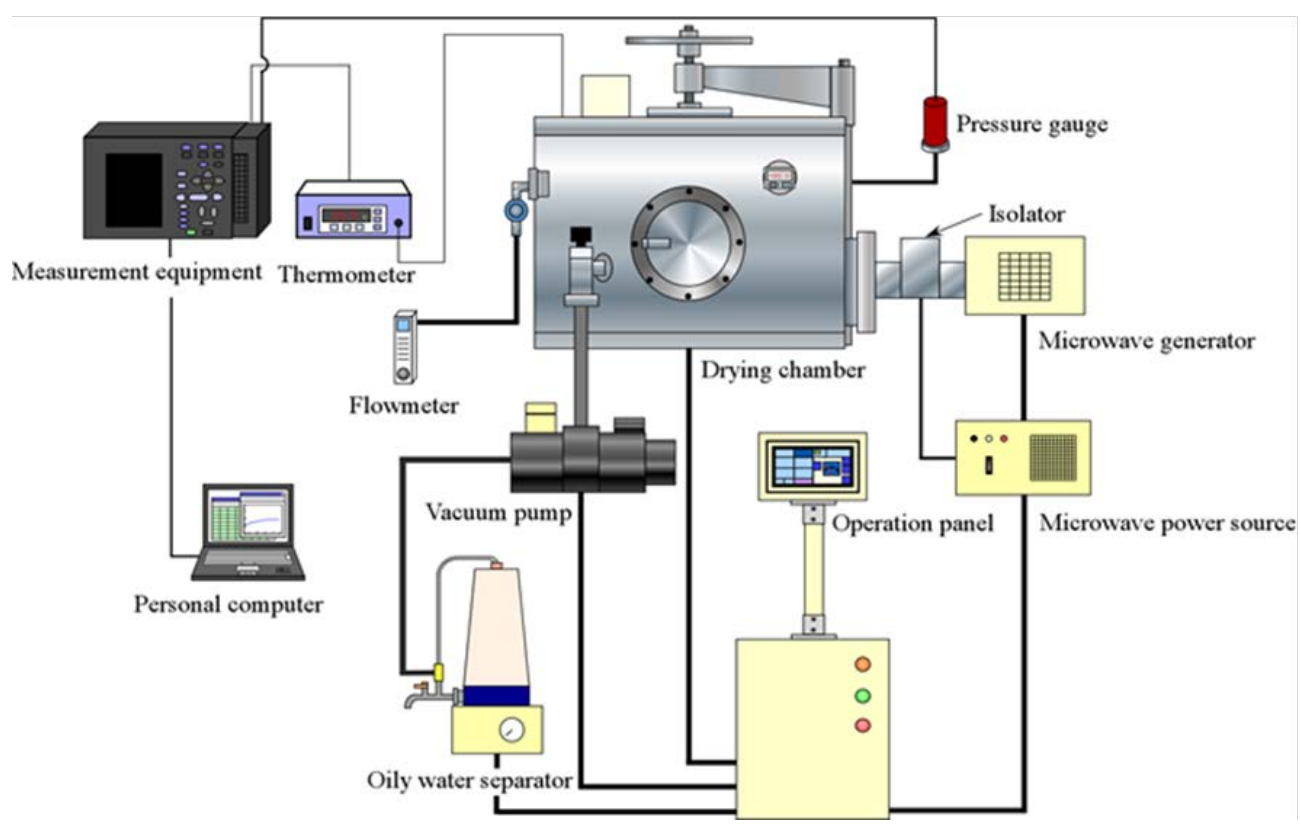

Fig.2 Microwave vacuum drying system. 


\subsection{Evaluation method of protein conformation changes}

Molecular structure analysis was performed using a circular dichroism spectrometer (CD; JASCO Corporation J-820) in order to confirm the denaturation of proteins by drying. Three kinds of dried egg white by MVD, FD and their combination were subjected to the tests. As a reference sample showing thermal damage, the egg white heated at $98{ }^{\circ} \mathrm{C}$ for 10 minutes was examined as well as the raw egg white. Ultraviolet visible spectrophotometer (Hitachi High-Tech Science U-3310) was used to adjust to the same concentration, and ultra pure water was used as a solvent.

\subsection{Measurement of the residual activity of protein}

It is most important to confirm the residual activity of the protein. Therefore, the degradation activity of lysozyme was measured using the UV spectrophotometer. Micrococcus luteus was added to $50 \mathrm{mM}$ phosphate buffer solution so that the absorbance at a wavelength of $600 \mathrm{~nm}$ was 1.0 Abs in a $1 \mathrm{~cm}$ glass cell to prepare a substrate solution. The aqueous solution of lysozyme of $20 \mu \mathrm{l}$ was dropped into $2980 \mu \mathrm{l}$ of the substrate solution, and the time change in absorbance at $600 \mathrm{~nm}$ due to substrate degradation of lysozyme was measured. The activity of lysozyme was evaluated assuming that the absorbance immediately after the dropping was $100 \%$ and the absorbance after the decomposition was $0 \%$.

\subsection{Solubility test}

Solubility is important factor for a practical use in medical area. In this study, $0.05 \mathrm{~g}$ of dried egg white was dissolved in $5 \mathrm{ml}$ of water and time change of concentration of albumin was measured.

\section{Results and Discussions}

\subsection{Drying of egg white}

Figure 3 shows appearances of egg white before and after the drying. The raw egg white is transparent as in (a), it is seen in (b) that dried egg white is a transparent film even after the MVD drying. On the other hand, in the FD of (c) and 1h-MVD+FD of (d), it became a porous state like a sponge cake. In the FD, since ice crystals are dried by sublimation, the ice crystal parts become voids and porous. In 1h-MVD+FD, it is considered that ice crystals became smaller by first 1-hour drying by the MVD, and the porosity decreased. Also, we can see many large cracks in (d). 


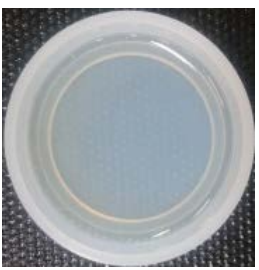

(a)

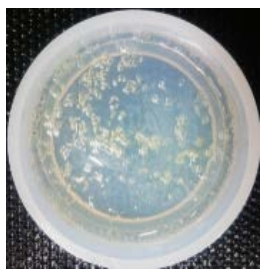

(b)

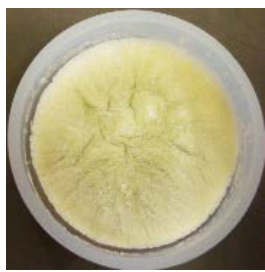

(c)

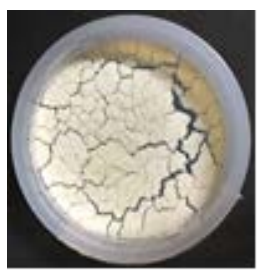

(d)

Fig.3 Photographs of egg white before and after drying.

(a) Raw white egg, (b) MVD, (c) FD, (d) 1h-MVD+FD

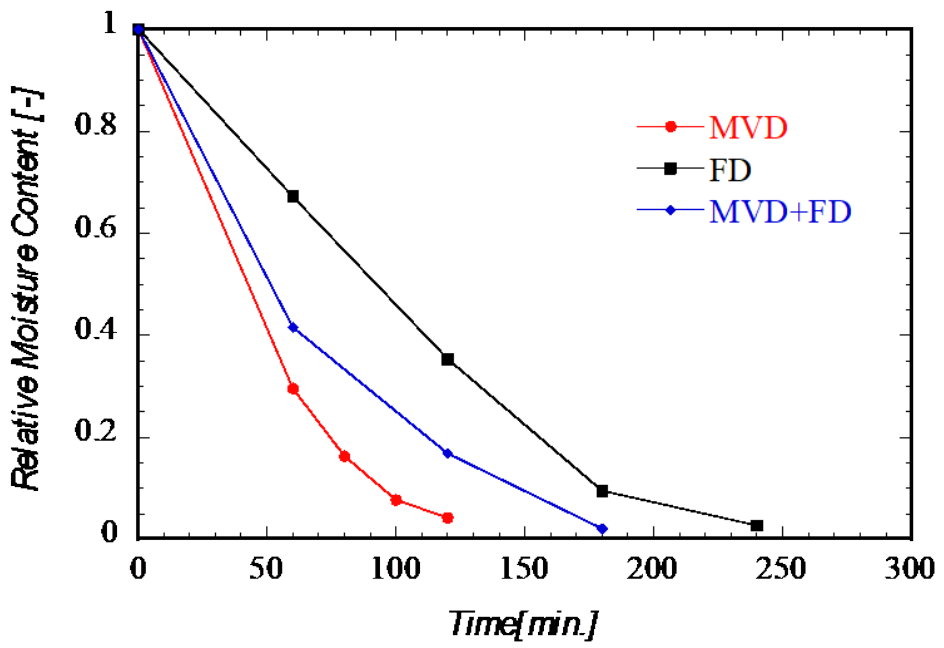

Fig.4 Time transients of moisture content ratio.

Figure 4 shows the time transient of the relative moisture content in each drying process. Drying was carried out until water contents became 0.05 or less. In MVD, the drying temperature was kept bellow $25{ }^{\circ} \mathrm{C}$. Compared to FD, MVD was able to reduce drying time by about $50 \%$ and MVD+FD by about $20 \%$.

\subsection{Molecular structure analysis of egg white (albumin)}

The results of structural analysis on dried egg albumin by CD are shown in Fig.5. It is known that the albumin has two negative peaks at $210 \mathrm{~nm}$ and $218 \mathrm{~nm}$ in the CD signal. The present results for FD, MVD and MVD+FD indicate two negative peaks similar to the raw egg albumin [6]. However, the CD intensity of the $98{ }^{\circ} \mathrm{C}$-heated sample decreased in wide ranges, and the negative peaks shifted to shorter wavelengths of $207 \mathrm{~nm}$ and $217 \mathrm{~nm}$, which indicates that alpha-helix structure of albumin has changed due to heating. It is 
confirmed from the results that the room temperature dryings can preserve the albumin without any structural damages.

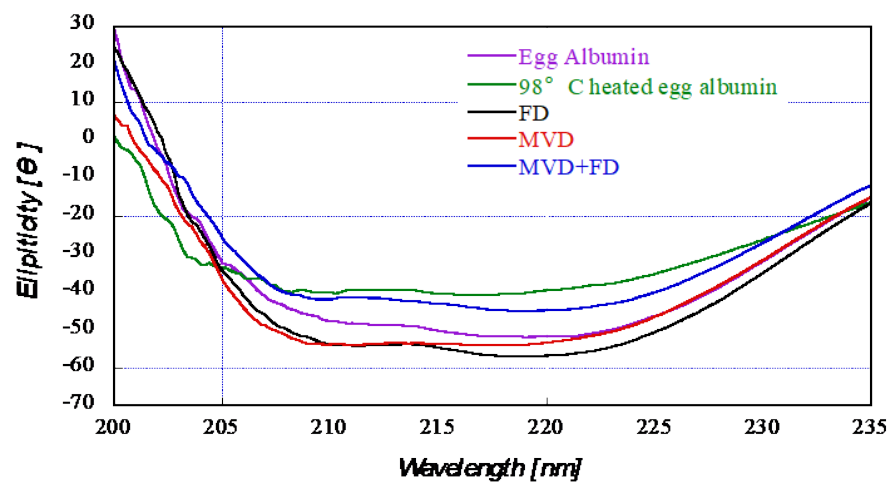

Fig.5 CD spectral analysis of albumin aqueous solution.

\subsection{Residual activity of lysozyme}

Figure 6 shows the residual activity of lysozyme measured using UV, where the absorbance at $600 \mathrm{~nm}$ of wave length was measured by dropping lysozyme into the substrate solution at 150 s. It can be seen that the absorbance of lysozyme heated at $98{ }^{\circ} \mathrm{C}$ has not changed. This is because lysozyme was inactivated and could not decompose micrococcus luthus. In contrast, lysozyme processed by FD, MVD, MVD+FD has decreased absorbance after 150s, indicating that the activity of degrading micrococcus leutus can be preserved. The results of calculating the decomposition rate are shown in Table 1 . There is no clear difference in the drying method compared to lysozyme.

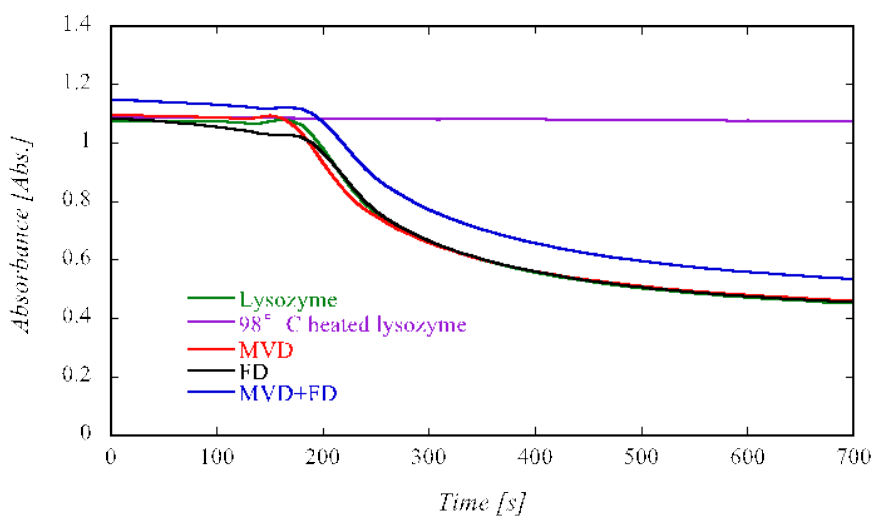

Fig.6 Remaining activity measurement of lysozyme aqueous solution. 
Table 1. Decomposition rate

\begin{tabular}{ccccc}
\hline & Lysozyme & MVD & FD & MVD+ FD \\
\hline Decomposition rate (Abs/s) & 0.003791 & 0.003924 & 0.003517 & 0.003192 \\
\hline
\end{tabular}

\subsection{Comparison of solubility}

Figure 7 compares the time transient of concentration when $0.05 \mathrm{~g}$ of dried egg white is dissolved in $5 \mathrm{ml}$ of ultrapure water. It can be seen that the sample by FD treatment dissolved in approximately 10 minutes, while the sample by MVD took about 40 minutes. About $80 \%$ or more of sample by MVD + FD has dissolved in the first 20 minutes and it can be said that it is more soluble than MVD. This indicates that ice crystal formation during the freezing contributes the faster solubility.

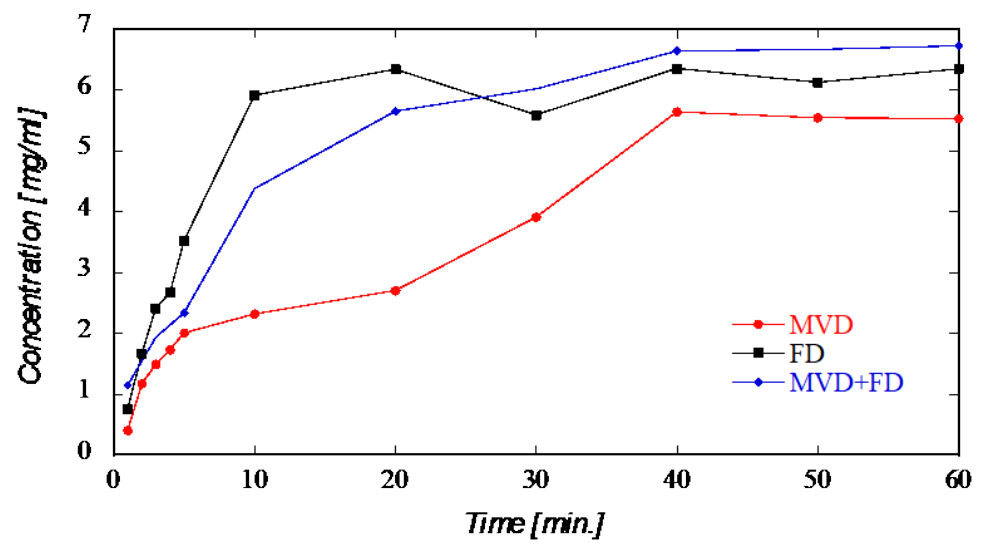

Fig.7 Comparison of solubility of MVD, FD and MVD+FD.

\section{Conclusions}

The protein aqueous solutions, egg white (albumin) and lysozyme, were dried using three kinds of drying methods, i.e. the freeze-drying (FD) and the microwave vacuum drying (MVD) and their combination, and the conformation change and the activity of preserved protein were examined. Circular dichroism (CD) spectrometer is used to detect protein conformation changes in the drying of albumin, which indicates that the MVD at room temperature can preserve the protein conformation. Also, the UV measurement of the residual activity of lysozyme with use of the micrococcus luteus shows that the room temperature drying shows good performance for the residual activity. It is concluded that the MVD at room temperature enables a less damages and a rapid drying compared to the 
FD. It should be noted that MVD is inferior in solubility compared with the FD. The combination of MVD and FD has a possibility for better performances in solubility and drying time.

\section{Acknowledgements}

CD analysis and chemical works were supported by Prof. Shigeori Takenaka, Department of Applied Chemistry, Kyushu Institute of Technology. This study was financially supported by the Ministry of Education, Science, Sports and Culture, Grant-in-Aid for Scientific Research, Project No. 17K18843.

\section{References}

[1] Vázquez-Rey, M.; Lang, D.A. Review; Aggregates in monoclonal antibody manufacturing processes. Biotechnology and Bioengineering 2011, 108 (7), 14941508.

[2] Mathias, S.F.; Franks, F.; Hatley, R.H.; The stabilization of proteins by freeze-drying and by alternative methods. In polypeptide and protein drugs: production, characterization and formulation; Hider, R.C, Barlow, D., Eds.; Ellis Horwood Limited.: West Sussex, 1991; 120-131.

[3] Franks, F. Improved freeze-drying: from empiricism to predictability. Cryo-Letters 1990, 11, 93-110.

[4] Tsuruta, T.; Hayashi, T. Enhancement of microwave drying under reduced pressure condition by irradiation control and external air supply. In Proceedings of the Third Nordic Drying Conference NDC2005, Karlstad, Sweden, June 15-17, 2005.

[5] Tsuruta, T.; Tanigawa, H.; Sashi, H. Study on shrinkage deformation of food in microwave-vacuum drying. Drying Technology 2015, 33(5), 1830-1836.

[6] Townend, R.; Kumosinski, T.F.; Timasheff, S.N.; Fasman, G.D.; Davidson, B. The circular dichroism of the $\beta$ structure of poly-l-lysine 1966 23(2), 163-169. 